\title{
EQUISYMMETRIC STRATA OF THE MODULI SPACE OF CYCLIC TRIGONAL RIEMANN SURFACES OF GENUS 4
}

\author{
MILAGROS IZQUIERDO* \\ Matematiska institutionen, Linköpings universitet, 58183 Linköping, Sweden \\ e-mail:miizq@mai.liu.se \\ and DANIEL YING** \\ Matematiska institutionen, Linköpings universitet, 58183 Linköping, Sweden \\ e-mail:dayin@mai.liu.se
}

(Received 12 December 2006; accepted 25 September 2008)

\begin{abstract}
A closed Riemann surface which can be realized as a three-sheeted covering of the Riemann sphere is called trigonal, and such a covering is called a trigonal morphism. If the trigonal morphism is a cyclic regular covering, the Riemann surface is called a cyclic trigonal Riemann surface. Using the characterization of cyclic trigonality by Fuchsian groups, we find the structure of the space of cyclic trigonal Riemann surfaces of genus 4.
\end{abstract}

1. Introduction. A closed Riemann surface $X$ which can be realized as a threesheeted covering of the Riemann sphere is said to be trigonal, and such a covering will be called a trigonal morphism. This is equivalent to the fact that $X$ is represented by a curve given by a polynomial equation of the form:

$$
y^{3}+y b(x)+c(x)=0 .
$$

If $b(x) \equiv 0$ then the trigonal morphism is a cyclic regular covering and the Riemann surface is called cyclic trigonal. Trigonal Riemann surfaces and their generalizations have been recently studied (see $[2,3,15]$ ).

By Lemma 2.1 of ref. [1], if the surface $X$ has genus $g \geq 5$ then the trigonal morphism is unique. The Severi-Castelnouvo inequality is used in order to prove such uniqueness, but this technique is not valid for small genera.

Using the characterization of trigonality by means of Fuchsian groups [6], we obtain all possible cyclic trigonal Riemann surfaces of genus 4 . The space of cyclic trigonal Riemann surfaces of genus 4 consists of two open balls of (complex-) dimension 3 (see [10]). The singularity of the space consists of the trigonal Riemann surfaces with automorphisms group containing the group generated by the trigonal morphism. These singularities are naturally stratified according to their full group of automorphisms. In this paper, we find the stratification of the moduli space of cyclic trigonal Riemann surfaces of genus 4 into smooth locally closed subvarieties, the equisymmetric strata [4] such that each stratum consists of equisymmetric surfaces. Two closed Riemann surfaces $X, \bar{X}$ of genus $g$ are called equisymmetric if their

\footnotetext{
*Partially supported by the Swedish Research Council (VR).

*** Results in this paper form part of Daniel Ying's PhD Thesis.
} 
automorphisms groups determine conjugate subgroups of the mapping class group of genus $g$. The equisymmetric strata are in one-to-one correspondence with topological equivalence classes of orientation-preserving actions of a finite group $G$ on a surface $X$. Two finite groups $G^{\prime} \leq G$ can induce the same stratum if they are quotient groups of a pair of Fuchsian groups $\Delta^{\prime} \leq \Delta$, where $\Delta^{\prime}$ is a non-maximal Fuchsian group [14].

2. Trigonal Riemann surfaces and Fuchsian groups. Let $X_{g}$ be a compact Riemann surface of genus $g \geq 2$. $X_{g}$ can be represented as a quotient $X_{g}=\mathcal{D} / \Gamma$ of the unit disc $\mathcal{D}$ under the action of a (cocompact) Fuchsian group $\Gamma$. The algebraic structure of $\Gamma$ and the geometric structure of $X_{g}=\mathcal{D} / \Gamma$ are given by the signature of $\Gamma$

$$
s(\Gamma)=\left(g ; m_{1}, \ldots, m_{r}\right) .
$$

Given a subgroup $\Gamma^{\prime}$ of index $N$ in a Fuchsian group $\Gamma$, one can calculate the structure of $\Gamma^{\prime}$ by Theorem 1 .

THEOREM 1. [13]. Let $\Gamma$ be a Fuchsian group with signature (1) and canonical presentation (2). Then $\Gamma$ contains a subgroup $\Gamma^{\prime}$ of index $N$ with signature

$$
s\left(\Gamma^{\prime}\right)=\left(h ; m_{11}^{\prime}, m_{12}^{\prime}, \ldots, m_{1 s_{1}}^{\prime}, \ldots, m_{r 1}^{\prime}, \ldots, m_{r s_{r}}^{\prime}\right),
$$

if and only if there exists a transitive permutation representation $\theta: \Gamma \rightarrow \Sigma_{N}$ satisfying the following conditions:

(1) The permutation $\theta\left(x_{i}\right)$ has precisely $s_{i}$ cycles of lengths less than $m_{i}$, the lengths of these cycles being $m_{i} / m_{i 1}^{\prime}, \ldots, m_{i} / m_{i s_{i}}^{\prime}$.

(2) The Riemann-Hurwitz formula

$$
\mu\left(\Gamma^{\prime}\right) / \mu(\Gamma)=N
$$

$\mu(\Gamma), \mu\left(\Gamma^{\prime}\right)$ the hyperbolic areas of the surfaces $\mathcal{D} / \Gamma, \mathcal{D} / \Gamma^{\prime}$, respectively.

Given a Riemann surface $X=\mathcal{D} / \Gamma$, with $\Gamma$ a surface Fuchsian group, a finite group $G$ is a group of automorphisms of $X$ if and only if there exists a Fuchsian group $\Delta$ and an epimorphism $\theta: \Delta \rightarrow G$ with $\operatorname{ker}(\theta)=\Gamma$.

Let $\Gamma$ be a Fuchsian group with signature (1). Then the Teichmüller space $T(\Gamma)$ of $\Gamma$ is homeomorphic to a complex ball of dimension $d(\Gamma)=3 g-3+r$ (see [12]). Let $\Gamma^{\prime} \leq \Gamma$ be Fuchsian group, the inclusion mapping $\alpha: \Gamma \rightarrow \Gamma^{\prime}$ induces an embedding $T(\alpha): T(\Gamma) \rightarrow T\left(\Gamma^{\prime}\right)$ defined by $[r] \mapsto[r \alpha]($ see $[12,14])$. The modular group $\operatorname{Mod}(\Gamma)$ of $\Gamma$ is the quotient $\operatorname{Mod}(\Gamma)=\operatorname{Aut}(\Gamma) / \operatorname{Inn}(\Gamma)$. The moduli space of $\Gamma$ is the quotient $M(\Gamma)=T(\Gamma) / \operatorname{Mod}(\Gamma)$ endowed with the quotient topology.

A Fuchsian group $\Gamma$ such that there does not exist any other Fuchsian group containing it with finite index is called a finite maximal Fuchsian group. To decide whether a given finite group can be the full group of automorphism of some compact Riemann surface, we will need all pairs of signatures $s(\Gamma)$ and $s\left(\Gamma^{\prime}\right)$ for some Fuchsian groups $\Gamma$ and $\Gamma^{\prime}$ such that $\Gamma^{\prime} \leq \Gamma$ and $d(\Gamma)=d\left(\Gamma^{\prime}\right)$. The full list of such pairs of signatures was obtained by Singerman in [14].

Definition 2. A Riemann surface $X$ is said to be trigonal if it admits a threesheeted covering $f: X \rightarrow \widehat{\mathbb{C}}$ onto the Riemann sphere. If $f$ is a cyclic regular covering then $X$ is called cyclic trigonal. The covering $f$ will be called the (cyclic) trigonal morphism. 
The following result gives us a characterization of cyclic trigonal Riemann surfaces using Fuchsian groups:

THEOREM 3. [6]. Let $X_{g}$ be a Riemann surface, $X_{g}$ admits a cyclic trigonal morphism $f$ if and only if there is a Fuchsian group $\Delta$ with signature $(0 ; \overbrace{3, \ldots, 3}^{g+2})$ and an index three normal surface subgroup $\Gamma$ of $\Delta$, such that $\Gamma$ uniformizes $X_{g}$.

By Lemma 2.1 of [1], if the surface $X_{g}$ has genus $g \geq 5$, then the trigonal morphism is unique. In this case, the cyclic trigonal morphism $f$ is induced by a normal subgroup $C_{3}$ in $\operatorname{Aut}\left(X_{g}\right)$ (see [10]).

Our aim is to show the structure of the space $\mathcal{M}_{4}^{3}$, its equisymmetric stratification. To do that we will find, by means of Fuchsian groups, the classes of actions of finite groups on cyclic trigonal surfaces $X_{4}$ of genus 4 .

3. Strata of cyclic trigonal Riemann surfaces of genus 4. Two closed Riemann surfaces $X, \bar{X}$ of genus 4 are called equisymmetric if their automorphisms groups determine conjugate subgroups of the mapping class group of genus 4 . The equisymmetric strata are in one-to-one correspondence with topological equivalence classes of orientation-preserving actions of a finite group $G$ on a surface $X$. Each (effective and orientable) action of a finite group $G$ on a surface $X_{4}$ is determined by an epimorphism $\theta: \Delta \rightarrow G$ from the Fuchsian group $\Delta$ such that $\operatorname{ker}(\theta)=\Gamma$, where $X_{4}=$ $\mathcal{D} / \Gamma$ and $\Gamma$ is a surface Fuchsian group. The group $\Delta$ has signature as in Lemma 4 . Two finite groups $G^{\prime}, G\left(G^{\prime} \leq G\right)$ can induce the same stratum if they are quotient groups of pair of Fuchsian groups $\Delta^{\prime} \leq \Delta$, where $\Delta^{\prime}$ is a non-maximal Fuchsian group [14]. In this case $G^{\prime}$ is not the full automorphisms group of the Riemann surface $X$.

Two actions $\epsilon, \epsilon^{\prime}$ of $G$ on a Riemann surface $X$ are (weakly) topologically equivalent if there is a $w \in \operatorname{Aut}(G)$ and an $h \in \operatorname{Hom}^{+}(X)$ such that $\epsilon^{\prime}(g)=h \epsilon w(g) h^{-1}$. Paraphrasing it in terms of groups: two epimorphisms $\theta_{1}, \theta_{2}: \Delta \rightarrow G$ define two topologically equivalent actions of $G$ on $X$ if there exist automorphisms $\phi: \Delta \rightarrow \Delta$, $w: G \rightarrow G$ such that $\theta_{2}=w \theta_{1} \phi^{-1}$. With other words, let $\mathcal{B}$ be the subgroup of $\operatorname{Aut}(\Delta)$ induced by orientation-preserving homeomorphisms. Then two different epimorphisms $\theta_{1}, \theta_{2}: \Delta \rightarrow G$ define the same class of $G$-actions if and only if they lie in the same $\mathcal{B} \times \operatorname{Aut}(G)$-class (see $[4,5,11]$ ).

We need now an algebraic characterization of $\mathcal{B}$. Since we are interested in trigonal Riemann surfaces, we consider the groups

$$
\bar{\Delta}=\left\langle\bar{x}_{1}, \bar{x}_{2}, \ldots, \bar{x}_{r} \mid \bar{x}_{1} \bar{x}_{2} \ldots \bar{x}_{r}=1\right\rangle .
$$

$\bar{\Delta}$ is the fundamental group of the punctured surface $X_{0}$ obtained by removing the $r$ branch points of the quotient Riemann sphere $X / G . \mathcal{B}$ can be identified with a certain subgroup of the mapping class group of $X_{0}$.

Now, any automorphism $\phi \in \Delta$ can be extended to an automorphism $\bar{\phi} \in \bar{\Delta}$ such that for $1 \leq j \leq r, \bar{\phi}\left(\bar{x}_{j}\right)$ is conjugate to some $\left(\bar{x}_{j}\right)$. The induced representation $\mathcal{B} \rightarrow \Sigma_{r}$ preserves the branching orders.

We are interested in finding elements of $\mathcal{B} \times \operatorname{Aut}(G)$ that make our epimorphisms $\theta_{1}, \theta_{2}: \Delta \rightarrow G$ equivalent. We can produce the automorphism $\phi \in \mathcal{B}$ ad hoc. In our case the only elements of $\mathcal{B}$ we need are compositions of $\phi_{i j}: x_{j} \rightarrow x_{j+1}$ and 
$x_{j+1} \rightarrow x_{j+1}^{-1} x_{j} x_{j+1}$, where we write down only the action on the generators moved by the automorphism $\phi_{i j}$.

We use Theorems 1 and 3 to find cyclic trigonal Riemann surfaces. Let $G$ be the full automorphisms group of the surface $X_{4}$.

Algorithm. Let $X_{4}=\mathcal{D} / \Gamma$ be a Riemann surface of genus 4 uniformized by the surface Fuchsian group $\Gamma, X_{4}$ admits a cyclic trigonal morphism $f$ if and only if there is a maximal Fuchsian group $\Delta$ with signature $\left(0 ; m_{1}, \ldots, m_{r}\right)$, a trigonal automorphism $\varphi$ : $X_{4} \rightarrow X_{4}$, such that $\langle\varphi\rangle \leq G$ and an epimorphism $\theta: \Delta \rightarrow G$ with $\operatorname{ker}(\theta)=\Gamma$ a surface group and such that $\theta^{-1}(\langle\varphi\rangle)$ is a Fuchsian group with signature $(0 ; 3,3,3,3,3,3)$.

REMARK. The condition $\Gamma$ to be a surface Fuchsian group imposes that the order of the image under $\theta$ of an elliptic generator $x_{i}$ of $\Delta$ is the same as the order of $x_{i}$ and $\theta\left(x_{1}\right) \theta\left(x_{2}\right) \ldots, \theta\left(x_{r-1}\right)=\theta\left(x_{r}\right)^{-1}$. Now, let $\theta: \Delta \rightarrow G$ be such an epimorphism and let $|G|=N$. Then $s(\Delta)=\left(0 ; m_{1}, \ldots, m_{r}\right)$, where $m_{i}$ runs over the divisors of $N$. Applying the Riemann-Hurwitz formula we have that

$$
2(g+N-1)=\sum_{1}^{r} N \frac{\left(m_{i}-1\right)}{m_{i}} .
$$

Equation (5) and the list of maximal signatures [14] yield the following list of allowed signatures for genus 4 . It is well-konown that a surface of genus 4 has automorphisms group of order divisible by 2,3 or 5 .

Lemma 4. Let $X_{4}=\mathcal{D} / \Gamma$ be a Riemann surface of genus 4 uniformized by the surface group $\Gamma, X_{4}$ admitting a cyclic trigonal morphism $f$. Then the Fuchsian group $\Delta$ uniformizing the orbifold $X / G$ must have one of the following signatures:

\begin{tabular}{clllll}
\hline$|G|$ & $s(\Delta)$ & $|G|$ & $s(\Delta)$ & $|G|$ & $s(\Delta)$ \\
\hline 3 & $(0 ; 3,3,3,3,3,3)$ & 6 & $(0 ; 2,6,6,6)$ & 6 & $(0 ; 2,2,3,3,3)$ \\
6 & $(0 ; 2,2,2,3,6)$ & 6 & $(0 ; 2,2,2,2,2,2)$ & 6 & $(0 ; 3,3,6,6)^{a}$ \\
9 & $(0 ; 9,9,9)^{a}$ & 9 & $(0 ; 3,3,3,3)^{a}$ & 12 & $(0 ; 4,6,12)$ \\
12 & $(0 ; 2,2,2,2,2)$ & 12 & $(0 ; 2,3,3,3)$ & 12 & $(0 ; 2,2,3,6)$ \\
12 & $(0 ; 6,6,6)^{a}$ & 12 & $(0 ; 3,12,12)^{a}$ & 12 & $(0 ; 2,2,4,4)^{a}$ \\
15 & $(0 ; 5,5,5)^{a}$ & 15 & $(0 ; 3,5,15)^{a}$ & 18 & $(0 ; 2,2,2,6)$ \\
18 & $(0 ; 3,6,6)^{a}$ & 18 & $(0 ; 2,9,18)^{a}$ & 18 & $(0 ; 2,2,3,3)^{a}$ \\
24 & $(0 ; 4,4,4)^{a}$ & 24 & $(0 ; 3,4,6)$ & 24 & $(0 ; 2,2,2,4)$ \\
24 & $(0 ; 2,6,12)^{a}$ & 24 & $(0 ; 3,3,12)^{a}$ & 24 & $(0 ; 2,8,8)^{a}$ \\
27 & $(0 ; 3,3,9)^{a}$ & 30 & $(0 ; 2,5,10)^{a}$ & 36 & $(0 ; 2,4,12)$ \\
36 & $(0 ; 2,2,2,3)$ & 36 & $(0 ; 3,3,6)^{a}$ & 36 & $(0 ; 3,4,4)^{a}$ \\
36 & $(0 ; 2,6,6)^{a}$ & 45 & $(0 ; 3,3,5)^{a}$ & 48 & $(0 ; 2,3,24)$ \\
48 & $(0 ; 2,4,8)^{a}$ & 54 & $(0 ; 2,3,18)$ & 60 & $(0 ; 2,3,15)$ \\
60 & $(0 ; 2,5,5)^{a}$ & 72 & $(0 ; 2,4,6)$ & 72 & $(0 ; 2,3,12)$ \\
72 & $(0 ; 3,3,4)^{a}$ & 90 & $(0 ; 2,3,10)$ & 108 & $(0 ; 2,3,9)$ \\
120 & $(0 ; 2,4,5)$ & 144 & $(0 ; 2,3,8)$ & & \\
\hline
\end{tabular}

${ }^{a}$ Non-maximal signature.

Using the notation of the Algorithm mentioned above, here we calculate all possible classes of epimorphisms $\theta: \Delta \rightarrow G$ inducing cyclic trigonal Riemann surfaces. 
We separate the cases according to the order of the group $G$. Observe that, if $\Delta^{\prime}$ is a nonmaximal Fuchsian group in the group $\Delta$ with no possible epimorphisms $\theta^{\prime}: \Delta^{\prime} \rightarrow G^{\prime}$, then there are no epimorphisms $\theta: \Delta \rightarrow G$. We use Theorem 1 to calculate $\theta^{-1}(\langle\varphi\rangle)$.

(1) $|G|=3$. There are two classes of epimorphisms $\theta: \Delta \rightarrow C_{3}=\left\langle a \mid a^{3}=1\right\rangle$ where $s(\Delta)=(0 ; 3,3,3,3,3,3): \theta_{1}\left(x_{2 i}\right)=a$ and $\theta_{1}\left(x_{2 i-1}\right)=a^{-1}, 1 \leq i \leq 3$, and $\theta_{2}\left(x_{i}\right)=a$, $1 \leq i \leq 6$.

The cyclic trigonal Riemann surfaces of genus 4 form a space $\mathcal{M}_{4}^{3}$ of (complex) dimension $d(\Delta)=3$. This space consists of two disconnected components $\mathcal{C}_{1}$ and $\mathcal{C}_{2}$. The connected component $\mathcal{C}_{1}$ is given by actions $\theta_{1}$, where half the stabilizers of the fixed points rotate in opposite directions. The component $\mathcal{C}_{2}$ is given by actions $\theta_{2}$, where the stabilizers of all the fixed points rotate in the same direction (see also [10]).

(2) $|G|=6$.

(i) First, consider the signature $s\left(\Delta_{1}\right)=(0 ; 2,6,6,6)$. There are epimorphisms $\theta: \Delta \rightarrow C_{6}=\left\langle a \mid a^{6}=1\right\rangle$. Applying Theorem 1 to $\theta^{-1}\left(\left\langle a^{2}\right\rangle\right)$ we obtain $s\left(\theta^{-1}\left(\left\langle a^{2}\right\rangle\right)\right)=(1 ; 3,3,3)$ and the surfaces $\mathcal{D} / \operatorname{Ker}(\theta)$ are not trigonal.

(ii) Signature $s\left(\Delta_{2}\right)=(0 ; 2,2,3,3,3)$. There are epimorphisms from $\Delta_{2}$ onto both $D_{3}$ and $C_{6}$. In each case, each of $\theta\left(x_{3}\right), \theta\left(x_{4}\right)$ and $\theta\left(x_{5}\right)$ induces two cone points in $\mathcal{D} / \operatorname{Ker}(\theta)$. Thus $s\left(\theta^{-1}\left(C_{3}\right)\right)=(0 ; 3,3,3,3,3,3)$, therefore these surfaces are trigonal. Now, there is one natural epimorphism $\theta: \Delta_{2} \rightarrow C_{6}=\left\langle a \mid a^{6}=1\right\rangle$. Finally, there is one class of epimorphisms $\theta: \Delta_{2} \rightarrow D_{3}=\left\langle a, s \mid a^{3}=s^{2}=(s a)^{2}=1\right\rangle$ equivalent to $\theta\left(x_{1}\right)=s, \theta\left(x_{2}\right)=$ sa, $\theta\left(x_{3}\right)=a, \theta\left(x_{4}\right)=a^{2}$ applying a suitable conjugation in $D_{3}$ and the elements $\phi_{2,3}^{2} \phi_{2,3}^{2} \phi_{3,4} \phi_{2,3}^{2} \in \mathcal{B}$. Therefore there are two strata, with (complex) dimension 2, of cyclic trigonal Riemann surfaces of genus 4 with six automorphisms: one stratum, lying in $\mathcal{C}_{1}$, is determined by the unique class of actions of $D_{3}$, the second stratum, in $\mathcal{C}_{2}$, is determined by the unique action of $C_{6}$.

(iii) Signature $s\left(\Delta_{3}\right)=(0 ; 2,2,2,3,6)$. There are epimorphisms $\theta: \Delta_{3} \rightarrow C_{6}$. By Theorem 1 the surfaces $\mathcal{D} / \operatorname{Ker}(\theta)$ are not trigonal since $s\left(\theta^{-1}\left(\left\langle a^{2}\right\rangle\right)\right)=$ $(1 ; 3,3,3)$.

(iv) The signature $s\left(\Delta_{4}\right)=(0 ; 2,2,2,2,2,2)$ does not induce trigonal surfaces since the orders of the elliptic elements of $\Delta_{4}$ are relative prime to 3 .

(v) Fuchsian groups with signature $s\left(\Delta_{5}\right)=(0 ; 3,3,6,6)$ are non-maximal. The epimorphisms $\bar{\theta}: \Delta_{5} \rightarrow C_{6}$ extend to epimorphisms $\theta: \Delta \rightarrow C_{6} \times C_{2}$ and $\theta: \Delta \rightarrow D_{6}$, where $s(\Delta)=(0 ; 2,2,3,6)$, which are studied in case 4(iii).

(3) $|G|=9$

(i) Fuchsian groups with signature $s\left(\Delta_{1}\right)=(0 ; 9,9,9)$ are non-maximal. The epimorphisms $\bar{\theta}: \Delta_{1} \rightarrow C_{9}$ extend to epimorphisms $\theta: \Delta \rightarrow C_{18}$, where $s(\Delta)=(0 ; 2,9,18)$.

(ii) Groups with signature $s\left(\Delta_{2}\right)=(0 ; 3,3,3,3)$ are non-maximal. The epimorphisms $\bar{\theta}: \Delta_{2} \rightarrow C_{3} \times C_{3}$ extend to epimorphisms $\theta: \Delta \rightarrow G_{18}$, where $s(\Delta)=(0 ; 2,2,3,3)$. These epimorphisms are studied in cases 6 (iv) and 10 (ii).

(4) $|G|=12$.

(i) Signature $s\left(\Delta_{1}\right)=(0 ; 4,6,12)$. There are equivalent epimorphisms $\theta: \Delta \rightarrow$ $C_{12}=\left\langle a \mid a^{12}=1\right\rangle$. Applying Theorem 1 we obtain $s\left(\theta^{-1}\left(\left\langle a^{4}\right\rangle\right)\right)=(1 ; 3,3,3)$ and the surface $\mathcal{D} / \operatorname{Ker}(\theta)$ is not trigonal. 
(ii) Signature $s\left(\Delta_{2}\right)=(0 ; 2,3,3,3)$. There are epimorphisms $\theta: \Delta_{2} \rightarrow A_{4}$ equivalent to $\theta\left(x_{1}\right)=s, \theta\left(x_{2}\right)=a, \theta\left(x_{3}\right)=a s$ and $\theta\left(x_{4}\right)=$ sas. Since any element of order 3 in $A_{4}$ leaves just one coset fixed when acting on the $\langle a\rangle$, $\langle s a\rangle,\langle a s\rangle$ or the $\langle s a s\rangle$-cosets, thus $\theta^{-1}\left(C_{3}\right)$ has signature $(1 ; 3,3,3)$ and the corresponding surfaces are not trigonal.

(iii) Signature $s\left(\Delta_{3}\right)=(0 ; 2,2,3,6)$. There are epimorphisms from $\Delta_{3}$ onto both $D_{6}$ and $C_{6} \times C_{2}$. In each case, $\theta\left(x_{3}\right)$ induces four cone points and $\theta\left(x_{4}\right)$ induces two cone points in $\mathcal{D} / \operatorname{Ker}(\theta)$. Thus, these surfaces are trigonal. Now, there is one epimorphism $\theta: \Delta_{3} \rightarrow C_{6} \times C_{2}$. There is, up to conjugation in $D_{6}$, one epimorphism $\theta: \Delta_{3} \rightarrow D_{6}=\langle a, s| a^{6}=s^{2}=$ $\left.(s a)^{2}=1\right\rangle$ defined by $\theta\left(x_{1}\right)=s, \theta\left(x_{2}\right)=s a^{3}, \theta\left(x_{3}\right)=a^{2}$. Therefore there are two strata, with (complex) dimension 1 , of cyclic trigonal Riemann surfaces of genus 4 with 12 automorphisms: one stratum, lying in $\mathcal{C}_{1}$, is determined by the action of $D_{6}$, the second stratum, in $\mathcal{C}_{2}$, is determined by the action of $C_{6} \times C_{2}$.

(iv) Signature $s\left(\Delta_{4}\right)=(0 ; 2,2,2,2,2)$ as in case 2 (iv).

(v) Fuchsian groups with signature $s\left(\Delta_{5}\right)=(0 ; 6,6,6)$ are non-maximal. The epimorphisms $\bar{\theta}: \Delta_{5} \rightarrow C_{6} \times C_{2}$ extend to epimorphisms $\theta: \Delta \rightarrow C_{3} \times$ $D_{4}$, where $s(\Delta)=(0 ; 2,6,12)$, and the epimorphism $\phi_{1}: \Lambda_{1} \rightarrow C_{3} \times A_{4}$, with $s\left(\Lambda_{1}\right)=(0 ; 3,3,6)$. This epimorphism is studied in case 15(i).

(vi) Groups with signature $s\left(\Delta_{6}\right)=(0 ; 3,12,12)$ are non-maximal. The epimorphisms $\bar{\theta}: \Delta_{6} \rightarrow C_{12}$ extend to epimorphisms $\theta: \Delta \rightarrow C_{3} \times D_{4}$, where $s(\Delta)=(0 ; 2,6,12)$, studied in case $7(\mathrm{vi})$.

(vii) The signature $s\left(\Delta_{7}\right)=(0 ; 2,2,4,4)$ as case 2 (iv).

(5) $|G|=15$.

(i) Signature $s\left(\Delta_{1}\right)=(0 ; 5,5,5)$. There is no epimorphism $\theta: \Delta_{1} \rightarrow C_{15}$.

(ii) Signature $s\left(\Delta_{2}\right)=(0 ; 3,5,15)$. There is, up to conjugation in $C_{15}$, one epimorphism $\theta: \Delta_{2} \rightarrow C_{15}$ defined by $\theta\left(x_{1}\right)=a^{5}, \theta\left(x_{2}\right)=a^{2}$. By Theorem $1, s\left(\theta^{-1}\left(C_{3}\right)\right)=(0 ; 3,3,3,3,3,3)$. There is a unique cyclic trigonal surface $T_{4}$ of genus 4 with $\operatorname{Aut}\left(T_{4}\right)=C_{15}$.

(6) $|G|=18$.

(i) Signature $s\left(\Delta_{1}\right)=(0 ; 2,2,2,6)$. There is no group of order 18 generated by three involutions containing elements of order 6 [9].

(ii) Signature $s\left(\Delta_{2}\right)=(0 ; 2,9,18)$. The epimorphism $\theta: \Delta_{2} \rightarrow C_{18}=\left\langle a \mid a^{18}\right\rangle$ is defined as $\theta\left(x_{1}\right)=a^{9}, \theta\left(x_{2}\right)=a^{2}$. By Theorem $1, \theta^{-1}\left(\left\langle a^{6}\right\rangle\right)$ has signature $(1 ; 3,3,3)$, inducing non-trigonal surfaces.

(iii) Groups with signature $s\left(\Delta_{3}\right)=(0 ; 3,6,6)$ are non-maximal. The epimorphisms $\bar{\theta}_{1}: \Delta_{3} \rightarrow C_{6} \times C_{3}$ and $\bar{\theta}_{2}: \Delta_{3} \rightarrow C_{3} \times D_{3}$ extend to an epimorphism $\theta: \Delta \rightarrow C_{6} \times D_{3}$, where $s(\Delta)=(0 ; 2,6,6)$. This epimorphism is studied in case $10(\mathrm{v})$.

There is an epimorphism $\bar{\theta}_{3}: \Delta_{3} \rightarrow C_{3} \times D_{3}$ which extends to an epimorphism $\bar{\phi}_{2}: \Delta \rightarrow D_{3} \times D_{3}$, with $s(\Delta)=(0 ; 2,6,6)$. This last epimorphism extends to the one studied in case 15(ii).

(iv) Signature $s\left(\Delta_{4}\right)=(0 ; 2,2,3,3)$. We consider epimorphisms $\theta_{1,2}: \Delta_{4} \rightarrow$ $C_{3} \times D_{3}, \theta_{1}\left(x_{1}\right)=s a^{i}, \theta_{1}\left(x_{2}\right)=s a^{j}, i \neq j \in\{0,1,2\}, \theta_{1}\left(x_{3}\right)=a^{i-j} b, \theta_{1}\left(x_{4}\right)=$ $b^{2} ; \theta_{2}\left(x_{1}\right)=s, \theta_{2}\left(x_{2}\right)=s a, \theta_{2}\left(x_{3}\right)=a b, \theta_{2}\left(x_{4}\right)=a b^{2}$ and $\theta_{3}: \Delta_{4} \rightarrow\left(C_{3} \times\right.$ $\left.C_{3}\right) \rtimes C_{2}=\left\langle a, b, s \mid a^{3}=b^{3}=s^{2}=(s a)^{2}=\left(s b^{2}\right)=[a, b]=1\right\rangle$ defined as $\theta_{3}\left(x_{1}\right)=s a, \theta_{3}\left(x_{2}\right)=s b, \theta_{3}\left(x_{3}\right)=a b, \theta_{3}\left(x_{4}\right)=b^{2}$. Epimorphisms $\theta_{2}$ and 
$\theta_{3}$ extend to epimorphisms $\theta: \Delta \rightarrow D_{3} \times D_{3}$, with $S(\Delta)=(0 ; 2,2,2,3)$ studied in case 10(ii).

Epimorphisms $\theta_{1}: \Delta_{4} \rightarrow C_{3} \times D_{3}$ induced one equisymmetric stratum. Now, by Theorem 1, $\theta_{1}\left(x_{3}\right)$ leaves no fixed points when acting on the $\langle b\rangle$ - or $\langle a\rangle$-cosets and three fixed points when acting on the $\langle a b\rangle$ - or $\left\langle a^{2} b\right\rangle$-cosets. Again $\theta_{1}\left(x_{4}\right)$ leaves six fixed points when acting on the $\langle b\rangle$-cosets, and no fixed points on the $\langle a\rangle$-, $\langle a b\rangle$ - or $\left\langle a^{2} b\right\rangle$-cosets. Then $s\left(\theta_{1}^{-1}(\langle b\rangle)\right)=(0 ; 3,3,3,3,3,3)$. Thus, the surfaces $\mathcal{D} / \operatorname{Ker}\left(\theta_{1}\right)$ form one stratum, in $\mathcal{C}_{2}$, of cyclic trigonal Riemann surfaces with unique central trigonal morphism.

(7) $|G|=24$.

(i) Signature $(0 ; 2,2,2,4)$ as in case 4 (vii).

(ii) Signature $s\left(\Delta_{2}\right)=(0 ; 3,4,6)$. There are epimorphisms $\theta: \Delta_{2} \rightarrow\langle 2,3,3\rangle=$ $Q \rtimes C_{3}=\left\langle a, s, t \mid a^{3}=t^{4}=s^{4}=(s t)^{4}=1, s^{2}=t^{2}, a^{2} s a=t, a^{2} t a=s t\right\rangle$, for instance $\theta\left(x_{1}\right)=s t a, \theta\left(x_{2}\right)=s$ and $\theta\left(x_{3}\right)=s^{2} a^{2}$. Now, the group $\langle 2,3,3\rangle$ contains just one conjugacy class of subgroups of order 3 , and one conjugacy class of elements of order 6 , with representatives sta and $s^{2} a^{2}$, respectively. The action of sta on the $\langle a\rangle$-cosets induces two fixed points, while the action of $s^{2} a^{2}$ yields one fixed point. Thus $s\left(\theta^{-1}(\langle a\rangle)\right)$ is $(1 ; 3,3,3)$ and the corresponding surface is not trigonal.

(iii) The signature $s\left(\Delta_{3}\right)=(0 ; 4,4,4)$ admits no epimorphism (see [9]).

(iv) The signature $s\left(\Delta_{4}\right)=(0 ; 3,3,12)$ admits no epimorphisms (see [9]).

(v) The signature $s\left(\Delta_{5}\right)=(0 ; 2,8,8)$ admits no epimorphism (see [9]).

(vi) Groups with signature $s\left(\Delta_{6}\right)=(0 ; 2,6,12)$ are non-maximal. The epimorphisms $\bar{\theta}: \Delta_{6} \rightarrow D_{4} \times C_{3}$ extend to epimorphisms $\theta: \Delta \rightarrow C_{3} \times$ $\Sigma_{4}$, where $s(\Delta)=(0 ; 2,3,12)$ that are studied in case $15(\mathrm{i})$.

(8) $|G|=27$. The signature $s(\Delta)=(0 ; 3,3,9)$ admits no epimorphisms.

(9) $|G|=30$. As case 5(i).

(10) $|G|=36$.

(i) Signature $(0 ; 2,4,12)$. There is no group of order 36 generated by elements of orders 2 and 4 with product of order 12 .

(ii) Fuchsian groups $\Delta_{2}$ with signature $(0 ; 2,2,2,3)$. The surfaces $\mathcal{D} / \operatorname{Ker}(\theta)$ form a connected uniparametric subvariety ${ }^{36} \mathcal{M}_{4}^{3}$ of $\mathcal{M}_{4}^{3}$, lying in $\mathcal{C}_{1}$, where $\theta: \Delta_{2} \rightarrow D_{3} \times D_{3}$ defined by $\theta\left(x_{1}\right)=s, \theta\left(x_{2}\right)=t b, \theta\left(x_{3}\right)=s t a$ and $\theta\left(x_{4}\right)=a^{2} b, D_{3} \times D_{3}=\langle a, b, s, t| a^{3}=b^{3}=s^{2}=t^{2}=[a, b]=[s, b]=$ $\left.[t, a]=(s a)^{2}=(t b)^{2}=1\right\rangle($ see $[7,8])$.

(iii) The signature $s\left(\Delta_{3}\right)=(0 ; 3,3,6)$ is studied in case 15(i).

(iv) The signature $s\left(\Delta_{4}\right)=(0 ; 3,4,4)$ is studied in case 15(ii).

(v) Groups with signature $s\left(\Delta_{5}\right)=(0 ; 2,6,6)$ are extensions of groups with signature $s(\Lambda)=(0 ; 3,6,6)$. First of all, the non-maximal groups with signature $(0 ; 2,6,6)$ are subgroups of groups $\Delta$ with signature $s(\Delta)=$ $(0 ; 2,4,6)$. They are studied in case 15 (ii).

The maximal group $\Delta_{5}$ with signature $(0 ; 2,6,6)$ is defined by an extension of both $\bar{\theta}_{1}: \Delta_{3} \rightarrow C_{6} \times C_{3}$ and $\bar{\theta}_{2}: \Delta_{3} \rightarrow C_{3} \times D_{3}$ in case 6(iii) to $\theta: \Delta_{5} \rightarrow C_{6} \times D_{3}=\langle a, b, s, t| a^{3}=b^{3}=s^{2}=t^{2}=(s t)^{2}=[a, b]=[s, b]=[t, a]=$ $\left.(s a)^{2}=[t, b]=1\right\rangle$ defined by $\theta\left(x_{1}\right)=s a^{2}, \theta\left(x_{2}\right)=t a b^{2}$ and $\theta\left(x_{3}\right)=s t a b$. Applying Theorem 1 to the action of $\theta\left(\Delta_{5}\right)$ on the $\langle b\rangle$-, $\langle a\rangle$-, $\langle a b\rangle$ - and $\left\langle a^{2} b\right\rangle$-cosets we obtain $s\left(\theta^{-1}(\langle b\rangle)\right)=(0 ; 3,3,3,3,3,3)$. Thus, the Riemann surface $Z_{4}=\mathcal{D} / \operatorname{Ker}(\theta)$ is a cyclic trigonal Riemann surface admitting a unique central trigonal morphism in the component $\mathcal{C}_{2}$ of $\mathcal{M}_{4}^{3}$. 
(11) $|G|=45$. As case 5(i).

(12) $|G|=48$.

(i) Signature $s\left(\Delta_{1}\right)=(0 ; 2,3,24)$ as case 7 (iv).

(ii) Signature $s\left(\Delta_{2}\right)=(0 ; 2,4,8)$ as cases 7(iii) and (17v) (see [14]).

(13) $|G|=54$. As case 8 (see $[\mathbf{1 4}]$ ).

(14) $|G|=60$.

(i) The signature $(0 ; 2,3,15)$ admits no epimorphism.

(ii) The signature $s\left(\Delta_{2}\right)=(0 ; 2,5,5)$ does not induce trigonal surfaces.

(15) $|G|=72$.

(i) Consider Fuchsian groups $\Delta_{1}$ with signature $(0 ; 2,3,12)$. There is one cyclic trigonal Riemann surface $X_{4}$ with unique trigonal morphism. $X_{4}$ is uniformized by $\operatorname{Ker} \theta_{1}$, where $\theta_{1}: \Delta_{1} \rightarrow \Sigma_{4} \times C_{3}$, with $\theta_{1}\left(x_{1}\right)=\bar{s}, \theta_{1}\left(x_{2}\right)=$ $a b, \theta_{1}\left(x_{3}\right)=a^{2} \bar{s} b$ (see [7]).

(ii) Consider Fuchsian groups $\Delta_{2}$ with signature $(0 ; 2,4,6)$. There is one cyclic trigonal Riemann surface $Y_{4}$ with two trigonal morphisms. $Y_{4}$ is uniformized by $\operatorname{Ker}_{2}$, where $\theta_{2}: \Delta_{2} \rightarrow\left(C_{3} \times C_{3}\right) \rtimes C_{4}$, with $\theta_{2}\left(x_{1}\right)=s$, $\theta_{2}\left(x_{2}\right)=t a, \theta_{2}\left(x_{3}\right)=s t b($ see [7]).

(iii) The signature $(0 ; 3,3,4)$ is as case 7(iii) (see [14]).

(16) $|G|=90$. As case 5(i).

(17) $|G|=108$. As case 8 (see [14]).

(18) $|G|=120$. As case 14 (ii).

(19) $|G|=144$. As cases 7(iii)) and (v) (see [14]).

We summarize the above results in the following:

REMARK 5. [7]. There is a connected uniparametric family of Riemann surfaces $X_{4}(\lambda)$ of genus 4 admitting two cyclic trigonal morphisms. The surfaces $X_{4}(\lambda)$ have $G=$ $\operatorname{Aut}\left(X_{4}(\lambda)\right)=D_{3} \times D_{3}$ and the quotient Riemann surfaces $X_{4}(\lambda) / G$ are uniformized by the Fuchsian groups $\Delta$ with signature $s(\Delta)=(0 ; 2,2,2,3)$.

THEOREM 6. The space $\mathcal{M}_{4}^{3}$ of cyclic trigonal Riemann surfaces of genus 4 form a disconnected subspace of dimension 3 of the moduli space $\mathcal{M}_{4} . \mathcal{M}_{4}^{3}$ is the union of $\mathcal{C}_{1}$ and $\mathcal{C}_{2}$.

(1) The subspace ${ }^{6} \mathcal{M}_{4}^{3}$, determined by the Fuchsian groups $\Delta^{\prime \prime}$ with $s\left(\Delta^{\prime \prime}\right)=$ $(0 ; 2,2,3,3,3)$, formed by Riemann surfaces of genus 4 with automorphisms group of order 6 is a disconnected space of dimension 2 consisting of two connected components $\mathcal{C}_{1}^{6}$ and $\mathcal{C}_{2}^{6}$ consisting of trigonal Riemann surfaces with automorphisms groups $D_{3}$ and $C_{6}$, respectively. $\mathcal{C}_{i}^{6} \subset \mathcal{C}_{i}$.

(2) The subspace ${ }^{12} \mathcal{M}_{4}^{3}$, determined by the Fuchsian groups $\Delta^{\prime}$ with $s\left(\Delta^{\prime}\right)=$ $(0 ; 2,2,3,6)$, formed by Riemann surfaces of genus 4 with automorphism group of order 12 is a disconnected space of dimension 1 consisting of two connected components $\mathcal{C}_{1}^{12}$ and $\mathcal{C}_{2}^{12}$ consisting of trigonal Riemann surfaces with automorphisms groups $D_{6}$ and $C_{12}$, respectively. $\mathcal{C}_{i}^{12} \subset \mathcal{C}_{i}$.

(3) There is one cyclic trigonal Riemann surface $T_{4}$ determined by the Fuchsian group $\Delta_{4}$ with $s\left(\Delta_{4}\right)=(0 ; 3,5,15)$ and automorphisms group $C_{15} . T_{4} \in \mathcal{C}_{2}$.

(4) The subspace ${ }^{18} \mathcal{M}_{4}^{3}$, determined by the Fuchsian groups $\Delta^{\prime \prime \prime}$ with $s\left(\Delta^{\prime \prime \prime}\right)=$ $(0 ; 2,2,3,3)$, formed by Riemann surfaces of genus 4 with automorphisms group of order 18 is a connected space of dimension 1 consisting of trigonal Riemann surfaces with automorphisms group $D_{3} \times C_{3} .{ }^{18} \mathcal{M}_{4}^{3} \subset \mathcal{C}_{2}$.

(5) The connected subspace ${ }^{36} \mathcal{M}_{4}^{3}$ of $\mathcal{M}_{4}^{3}$ formed by Riemann surfaces $X_{4}(\lambda)$ of genus 4 with automorphisms group of order 36 is a space of dimension 1 determined by 
the Fuchsian groups $\Delta$ with $s(\Delta)=(0 ; 2,2,2,3)$. The automorphisms group of the Riemann surfaces is $D_{3} \times D_{3}$ and the surfaces admit two trigonal morphisms. ${ }^{36} \mathcal{M}_{4}^{3} \subset \mathcal{C}_{1}$.

(6) There is one cyclic trigonal Riemann surface $Z_{4}$ determined by the Fuchsian group $\Delta_{3}$ with $s\left(\Delta_{3}\right)=(0 ; 2,6,6)$ and automorphisms group $C_{6} \times D_{3} . Z_{4} \in \mathcal{C}_{2}$.

(7) There are exactly two cyclic trigonal Riemann surfaces $X_{4}$ and $Y_{4}$ of genus 4 with automorphisms groups of order 72.

(i) $X_{4}$ has one cyclic central trigonal morphism, Aut $\left(X_{4}\right)=\Sigma_{4} \times C_{3}$ and $X_{4} / \Sigma_{4} \times$ $C_{3}$ uniformized by the Fuchsian group $\Delta_{1}$ with $s\left(\Delta_{1}\right)=(0 ; 2,3,12) . X_{4} \in \mathcal{C}_{2}$.

(ii) $Y_{4}$ has two trigonal morphisms, $\operatorname{Aut}\left(Y_{4}\right)=\left(C_{3} \times C_{3}\right) \rtimes D_{4}$ and $Y_{4} /\left(C_{3} \times\right.$ $\left.C_{3}\right) \rtimes D_{4}$ uniformized by the Fuchsian group $\Delta_{2}$ with $s\left(\Delta_{2}\right)=(0 ; 2,4,6) . Y_{4} \in \mathcal{C}_{1}$.

The strata ${ }^{18} \mathcal{M}_{4}^{3},{ }^{36} \mathcal{M}_{4}^{3}, \mathcal{C}_{1}^{12}$ and $\mathcal{C}_{2}^{12}$ are punctured Riemann surfaces.

Furthermore, the structure of the space $\mathcal{M}_{4}^{3}$ is given in the following theorem. We find the inclusion relations between the different strata in the equisymmetric stratification of $\mathcal{M}_{4}^{3}$.

THEOREM 7. The different equisymmetric strata in Theorem 6 satisfy the following inclusion relations:

(1) The space ${ }^{12} \mathcal{M}_{4}^{3}$ is a subspace of ${ }^{6} \mathcal{M}_{4}^{3}$. Furthermore $\mathcal{C}_{i}^{12} \subset \mathcal{C}_{i}^{6}, i=1,2$.

(2) The space ${ }^{18} \mathcal{M}_{4}^{3}$ is a subspace of $\mathcal{C}_{2}^{6} \subset{ }^{6} \mathcal{M}_{4}^{3}$.

(3) The space ${ }^{36} \mathcal{M}_{4}^{3}$ is a subspace of $\mathcal{C}_{1}^{6} \subset{ }^{6} \mathcal{M}_{4}^{3}$.

(4) The surface $Z_{4}$ given in Theorem 6 (case 6) belongs to ${ }^{18} \mathcal{M}_{4}^{3} \cap \mathcal{C}_{2}^{12}$

(5) The surface $X_{4}$ given in Theorem 6 (case 7(i)) belongs to the space ${ }^{12} \mathcal{M}_{4}^{3}$

(6) The surface $Y_{4}$ given in Theorem 6 (case $7\left(\right.$ ii) ) belongs to ${ }^{36} \mathcal{M}_{4}^{3} \cap \mathcal{C}_{1}^{12}$.

Proof.

(1) In fact the Riemann surfaces $\mathcal{D} / \Delta^{\prime \prime}$ with $s\left(\Delta^{\prime \prime}\right)=(0 ; 2,2,3,3,3)$ are double coverings of the the Riemann surfaces $\mathcal{D} / \Delta^{\prime}$ with $s\left(\Delta^{\prime}\right)=(0 ; 2,2,3,6)$. Consider the map $\phi^{\prime \prime}: \Delta^{\prime} \rightarrow \Sigma_{2}$ defined by $\phi^{\prime \prime}\left(x_{1}^{\prime}\right)=(1,2), \phi^{\prime \prime}\left(x_{2}^{\prime}\right)=\phi^{\prime \prime}\left(x_{3}^{\prime}\right)=$ $1_{d}, \phi^{\prime \prime}\left(x_{4}^{\prime}\right)=(1,2)$. By Theorem $1, \phi^{\prime \prime}\left(x_{1}^{\prime}\right)$ induces no cone points, $\phi^{\prime \prime}\left(x_{2}^{\prime}\right)$ induces two cone points of order $2, \phi^{\prime \prime}\left(x_{3}^{\prime}\right)$ two cone points of order 3 and $\phi^{\prime \prime}\left(x_{4}^{\prime}\right)$ one of order 3. Thus $\phi^{\prime \prime}$ is the required monodromy of the covering $\mathcal{D} / \Delta^{\prime \prime} \rightarrow \mathcal{D} / \Delta^{\prime}$, with $\Delta^{\prime \prime}=\phi^{\prime \prime-1}(S t b(1))$.

(2) In fact the Riemann surfaces $\mathcal{D} / \Delta^{\prime \prime}$ with $s\left(\Delta^{\prime \prime}\right)=(0 ; 2,2,3,3,3)$ are threesheeted coverings of the the Riemann surfaces $\mathcal{D} / \Delta^{\prime \prime \prime}$ with $s\left(\Delta^{\prime \prime \prime}\right)=$ $(0 ; 2,2,3,3)$. Consider the map $\phi^{\prime \prime \prime}: \Delta^{\prime \prime \prime} \rightarrow \Sigma_{3}$ defined by $\phi^{\prime \prime \prime}\left(x_{1}^{\prime \prime \prime}\right)=(1,2)$, $\phi^{\prime \prime \prime}\left(x_{2}^{\prime \prime \prime}\right)=(2,3), \phi^{\prime \prime \prime}\left(x_{3}^{\prime \prime \prime}\right)=1_{d}, \phi^{\prime \prime \prime}\left(x_{4}^{\prime \prime \prime}\right)=(1,2,3)$. By Theorem $1, \phi^{\prime \prime \prime}\left(x_{1}^{\prime \prime \prime}\right)$ and $\phi^{\prime \prime \prime}\left(x_{2}^{\prime \prime \prime}\right)$ induce one cone point of order two each, $\phi^{\prime \prime \prime}\left(x_{3}^{\prime \prime \prime}\right)$ induces three cone points of order 3 and $\phi^{\prime \prime \prime}\left(x_{4}^{\prime \prime \prime}\right)$ no cone point. Thus, $\phi^{\prime \prime \prime}$ is the required monodromy of the covering $\mathcal{D} / \Delta^{\prime \prime} \rightarrow \mathcal{D} / \Delta^{\prime \prime \prime}$, with $\Delta^{\prime \prime}=\phi^{\prime \prime \prime}-1(\operatorname{Stb}(1))$. The monodromy $\phi^{\prime \prime \prime}$ yields the action of $C_{3} \times D_{3}$ on the $\langle b, s\rangle$-cosets, where $b, s$ as in case 6(iv).

(3) In fact the Riemann surfaces $\mathcal{D} / \Delta^{\prime \prime}$ with $s\left(\Delta^{\prime \prime}\right)=(0 ; 2,2,3,3,3)$ are six-sheeted coverings of the Riemann surfaces $\mathcal{D} / \Delta$ with $s(\Delta)=(0 ; 2,2,2,3)$. Consider the maps $\phi_{1}: \Delta \rightarrow \Sigma_{2}$ defined by $\phi_{1}\left(x_{1}\right)=\phi_{1}\left(x_{2}\right)=(1,2), \phi_{1}\left(x_{3}\right)=\phi_{1}\left(x_{4}\right)=1_{d}$ and $\phi_{2}: \Lambda \rightarrow \Sigma_{3}$ defined by $\phi_{2}\left(y_{1}\right)=(1,2), \phi_{2}\left(y_{2}\right)=(1,3), \phi_{2}\left(y_{3}\right)=(1,2,3)$, $\phi_{2}\left(y_{4}\right)=1_{d}$, where $\Lambda=\phi_{1}^{-1}(S t b(1))$. By Theorem $1, s(\Lambda)=(0 ; 2,2,3,3)$. Now $\phi_{2}\left(y_{1}\right)$ and $\phi_{2}\left(y_{2}\right)$ induce one cone point of order 2 each, and $\phi_{2}\left(y_{4}\right)$ induces three cone points of order 3 . Therefore the composition map $\phi_{2} \cdot \phi_{1}$ is the 
required monodromy of the covering $\mathcal{D} / \Delta^{\prime \prime} \rightarrow \mathcal{D} / \Delta$. Again $\Delta^{\prime \prime}=\phi_{2}^{-1}(\operatorname{Stb}(1))$. The monodromy $\phi_{2} \cdot \phi_{1}$ yields the action of $D_{3} \times D_{3}$ on the $\langle a b, s t\rangle$-cosets, where $a b$, st are defined in case 10(ii).

The space ${ }^{36} \mathcal{M}_{4}^{3}$ belongs to the subvariety $\mathcal{C}_{1}^{6} \subset{ }^{6} \mathcal{M}_{4}^{3}$ consisting of the Riemann surfaces with half the stabilizers of the cone points rotating in opposite directions since $\langle a b, s t\rangle=D_{3}$.

(4) First of all, a Riemann surface $\mathcal{D} / \Delta^{\prime}$ with $s\left(\Delta^{\prime}\right)=(0 ; 2,2,3,6)$ is a threesheeted covering of the Riemann surface $Z_{4}=\mathcal{D} / \Delta_{3}$ with $s\left(\Delta_{3}\right)=(0 ; 2,6,6)$. Consider the representation $\phi: \Delta_{2} \rightarrow \Sigma_{3}$ defined by $\phi\left(\bar{y}_{1}\right)=(1,2), \phi\left(\bar{y}_{2}\right)=$ $(2,3), \phi\left(\bar{y}_{3}\right)=(1,2,3)$. By Theorem $1, \phi\left(\bar{y}_{1}\right)$ induces one cone point of order 2 , $\phi\left(\bar{y}_{2}\right)$ induces one cone point of order 3 and one cone point of order 6 and $\phi\left(\bar{y}_{3}\right)$ induces one cone point of order 2 . Then $s\left(\Delta^{\prime}\right)=s\left(\phi^{-1}(S t b(1))\right)=(0 ; 2,2,3,6)$. Thus, the map $\phi$ is the required monodromy of the covering $\mathcal{D} / \Delta^{\prime} \rightarrow \mathcal{D} / \Delta_{3}$. The monodromy $\phi$ yields the action of $C_{6} \times D_{3}$ on the $C_{6} \times C_{2}$-cosets, where $C_{6} \times C_{2}=\langle b, s, t\rangle$ as in case $10(\mathrm{v})$.

Secondly, a Riemann surface $\mathcal{D} / \Delta^{\prime \prime \prime}$ with $s\left(\Delta^{\prime \prime \prime}\right)=(0 ; 2,2,3,3)$ is a double covering of the the Riemann surface $Z_{4}$. By Theorem 1, the map $\tau: \Delta \rightarrow \Sigma_{2}$ defined by $\tau\left(\bar{x}_{1}\right)=1_{d}, \tau\left(\bar{x}_{2}\right)=\tau\left(\bar{x}_{3}\right)=(1,2)$ is the required monodromy of the covering $\mathcal{D} / \Delta^{\prime \prime \prime} \rightarrow \mathcal{D} / \Delta_{3}$ with $\Delta=\tau^{-1}(\operatorname{Stb}(1))$. Notice that $\tau\left(\bar{x}_{1}\right)$ induces two cone points of order 2 , and $\tau\left(\bar{x}_{2}\right)$ and $\tau\left(\bar{x}_{3}\right)$ one cone point of order 3 each. The monodromy $\tau$ yields the action of $C_{6} \times D_{3}$ on the $C_{3} \times D_{3}$-cosets, where $C_{3} \times D_{3}=\langle a, b, s\rangle$ as in case $10(\mathrm{v})$.

(5) A Riemann surface $\mathcal{D} / \Delta^{\prime}$ with $s\left(\Delta^{\prime}\right)=(0 ; 2,2,3,6)$ is a six-sheeted covering of the Riemann surface $\mathcal{D} / \Delta_{1}$ with $s\left(\Delta_{1}\right)=(0 ; 2,3,12)$. Consider the representation $\phi: \Delta_{1} \rightarrow \Sigma_{6}$ defined by $\phi\left(\bar{x}_{1}\right)=(2,4)(3,5), \phi\left(\bar{x}_{2}\right)=$ $(1,2,3)(4,5,6), \phi\left(\bar{x}_{3}\right)=(1,5,6,2)(3,4)$. By Theorem $1, \phi\left(\bar{x}_{1}\right)$ induces two cone points of order $2, \phi\left(\bar{x}_{2}\right)$ induces no cone points and $\phi\left(\bar{x}_{3}\right)$ induces one cone point of order 3 and one of order 6 , then $s\left(\Delta^{\prime}\right)=s\left(\phi^{-1}(S t b(1))\right)=(0 ; 2,2,3,6)$. Thus, the map $\phi$ is the required monodromy of the covering $\mathcal{D} / \Delta^{\prime} \rightarrow \mathcal{D} / \Delta_{1}$. The monodromy $\phi$ yields the action of $\Sigma_{4} \times C_{3}$ on the $C_{6} \times C_{2}$-cosets, where $C_{6} \times C_{2}=\left\langle b, \bar{s},(a \bar{s})^{2}\right\rangle$ as in case 15(i).

(6) First of all, a Riemann surface $\mathcal{D} / \Delta^{\prime}$ with $s\left(\Delta^{\prime}\right)=(0 ; 2,2,3,6)$ is a six-sheeted covering of the Riemann surface $Y_{4}=\mathcal{D} / \Delta_{2}$ with $s\left(\Delta_{2}\right)=(0 ; 2,4,6)$. Consider the representation $\phi: \Delta_{2} \rightarrow \Sigma_{6}$ defined by $\phi\left(\bar{y}_{1}\right)=(1,4)(2.3)(5,6), \phi\left(\bar{y}_{2}\right)=$ $(2,4,5,6)(1,3), \phi\left(\bar{y}_{3}\right)=(1,2,5)(3,4)$. By Theorem $1, \phi\left(\bar{y}_{1}\right)$ induces no cone points, $\phi\left(\bar{y}_{2}\right)$ induces one cone point of order 2 and $\phi\left(\bar{y}_{3}\right)$ induces one cone point of order 3 , one cone point of order 6 and one of order 2 , then $s\left(\Delta^{\prime}\right)=$ $s\left(\phi^{-1}(S t b(1))\right)=(0 ; 2,2,3,6)$. Thus, the map $\phi$ is the required monodromy of the covering $\mathcal{D} / \Delta^{\prime} \rightarrow \mathcal{D} / \Delta_{2}$. The monodromy $\phi$ yields the action of $\left(C_{3} \times\right.$ $\left.C_{3}\right) \rtimes D_{4}$ on the $D_{6}$-cosets, where $D_{6}=\left\langle a, s, t^{2}\right\rangle$ as in case 15 (ii).

Secondly, a Riemann surface $\mathcal{D} / \Delta$ with $s(\Delta)=(0 ; 2,2,2,3)$ is a double covering of the the Riemann surface $Y_{4}=\mathcal{D} / \Delta_{2}$ with $s\left(\Delta_{2}\right)=(0 ; 2,4,6)$. By Theorem 1 , the map $\tau: \Delta \rightarrow \Sigma_{2}$ defined by $\tau\left(\bar{x}_{1}\right)=1_{d}, \tau\left(\bar{x}_{2}\right)=\tau\left(\bar{x}_{3}\right)=(1,2)$ is the required monodromy of the covering $\mathcal{D} / \Delta \rightarrow \mathcal{D} / \Delta_{2}$ with $\Delta=\tau^{-1}(\operatorname{Stb}(1))$. Notice that $\tau\left(\bar{x}_{1}\right)$ induces two cone points of order $2, \tau\left(\bar{x}_{2}\right)$ the third cone point of order 2 and $\tau\left(\bar{x}_{3}\right)$ one cone point of order 3 . The monodromy $\tau$ yields the action of $\left(C_{3} \times C_{3}\right) \rtimes D_{4}$ on the $D_{3} \times D_{3}$-cosets, where $D_{3} \times D_{3}=\left\langle a, b, s, t^{2}\right\rangle$ as in case 15 (ii). 
REMARK. The Riemann surfaces $\mathcal{D} / \Delta^{\prime}$ with $s\left(\Delta^{\prime}\right)=(0 ; 2,2,3,6)$ cannot be coverings of the the Riemann surfaces $\mathcal{D} / \Delta$ with $s(\Delta)=(0 ; 2,2,2,3)$. Hence the space ${ }^{36} \mathcal{M}_{4}^{3}$ is not a subspace of $\mathcal{C}_{2}^{12} \subset{ }^{12} \mathcal{M}_{4}^{3}$.

The surface $X_{4}$ does not belong to ${ }^{18} \mathcal{M}_{4}^{3}$ since the group $C_{3} \times D_{3}$ is not a subgroup of the group $\Sigma_{4} \times C_{3}$.

\section{REFERENCES}

1. R. D. M. Accola, On cyclic trigonal Riemann surfaces, I, Trans. Am. Math. Soc. 283 (1984), 423-449.

2. R. D. M. Accola, A classification of trigonal Riemann surfaces, Kodai Math. J. 23 (2000), 81-87.

3. R. D. M. Accola, On the Castelnuovo-Severi inequality for Riemann surfaces, Kodai Math. J. 29 (2006), 299-317.

4. A. Broughton, The equisymmetric stratification of the moduli space and the Krull dimension of mapping class groups, Topol. Appl. 37 (1990), 101-113.

5. A. Broughton, Classifying finite group actions on surfaces of low genus, J. Pure Appl. Algebra 69 (1990), 233-270.

6. A. F. Costa and M. Izquierdo, On real trigonal Riemann surfaces, Math. Scand. 98 (2006), 53-468.

7. A. F. Costa, M. Izquierdo and D. Ying, On trigonal Riemann surfaces with non-unique morphisms, Manuscr. Math. 118 (2005), 443-453.

8. A. F. Costa, M. Izquierdo and D. Ying, On the family of cyclic trigonal Riemann surfaces of genus 4 with several trigonal morphisms, RACSAM, 101 (2007), 81-86.

9. H. S. M. Coxeter and W. O. J. Moser, Generators and relations for discrete groups (Springer-Verlag, Berlin, 1957).

10. G. González-Díez, On prime Galois covering of the Riemann sphere, Ann. Mat. Pure Appl. 168 (1995), 1-15.

11. W. Harvey, On branch loci in Teichmüller space, Trans. Am. Math. Soc. 153 (1971), 387-399.

12. S. Nag, The complex analytic theory of Teichmüller spaces (Wiley-Interscience Publication, NY, 1988).

13. D. Singerman, Subgroups of Fuchsian groups and finite permutation groups, Bull. London Math. Soc. 2 (1970), 319-323.

14. D. Singerman, Finitely maximal Fuchsian groups. J. Lond. Math. Soc. 6 (1972), 29-38.

15. A. Wootton, Non-normal Belyi p-gonal surfaces. Computational aspects of algebraic curves, 95-108, Lecture Notes Ser. Comput., 13, (World Sciientific Publications, Hackensack, NJ, 2005). 